\title{
The Bio-Mechanic Workspace plugin: enabling biomechanical measurement and simulation across workflows and software applications
}

\author{
Simon M. Harrison and Raymond C. Z. Cohen \\ CSIRO Data61, Clayton VIC Australia \\ Email: Simon.Harrison@csiro.au
}

\begin{abstract}
The Bio-Mechanic (BM) plugin for the CSIRO Workspace platform is presented and its benefits are discussed in context with the design principles and strengths of Workspace. The BM plugin facilitates a number of activities for biomechanics science including movement measurement, model personalisation, kinematic analysis, forward dynamics simulation, numerical analysis and visualisation preprocessing. Since this codebase is written as a plugin for Workspace it inherits by design a large range of interoperability, reusability and productivity benefits.
\end{abstract}

The structure of the BM plugin is presented in context with typical uses of the operations. File readers have been developed which populate model components from popularly used sources (e.g. common simulation package and marker-based motion capture formats). A range of operations can be used to clean input data, such as to reduce noise in movement or force data. Models can be scaled or rigged to surface meshes. Kinematic analysis can be carried out to determine the characteristics of a body movement or the differences between multiple movements. Forward dynamic simulation can be performed to determine the environmental loading on the body and the internal biomechanical loading such as tendon, muscle and joint forces. Results from kinematic analysis or forward simulation can be graphed using custom widgets and visualised in 3D. Operations and workflows are naturally interoperable, using the same datatypes and interfaces, and easily shareable. When combined with a user interface the workflows can be easily developed into standalone software programs.

Four use cases are presented to highlight the benefits of producing biomechanical workflows using the BM Workspace plugin. First, a dataset from the literature is visualised in $3 \mathrm{D}$ and the pose of the skeleton and muscles is easily displayed at any of the time instances of the supplied motion data. Second, a simple workflow is presented which uses the same dataset to simulate foot-ground contact during running and to predict the external force on the body and the resulting movement of the body centre of mass. This is a typical high value usage of simulation for biomechanics which is extremely easy to implement and customise for users without a software design background. Third, the use of the plugin to build, analyse, visualise and report on a large and detailed simulation that uses an external solver is described. A coupled biomechanicalsmoothed particle hydrodynamics ( $\mathrm{SPH}$ ) simulation is used to study sporting technique improvement where physical experimentation is difficult to perform and quantify. The plugin enables quick model development and analysis which is critical for timely completion of such large simulations. Fourth, a deployed software application built upon the BM plugin is described which combines a database of elite divers and more than sixty diving performances in an interactive virtual experimentation tool for coaches to improve springboard diving technique. Because the software was built in the Workspace environment it is modular, highly interoperable and its design was easily experimented with. As a result, its development was highly agile and productive.

Typical with Workspace plugins, the BM plugin has many benefits inherited from the use of the Workspace environment. The operations, workflows and standalone software are highly interactive and intuitive. Operations and workflows are naturally interoperable and reusable. Linkages with other code bases and solvers is easily achieved and already in place for many popular libraries. Productivity of users is increased by the aforementioned benefits which enables more breadth and depth of scientific inquiry to be completed. Finally, commercialisation and speed to market are improved because the method of use is efficient, restructuring can be done easily and quickly and because workflows can be converted into standalone software with little user effort.

Keywords: Biomechanics, simulation, Workspace, workflow, motion capture 
Harrison and Cohen, The Bio-Mechanic Workspace plugin: enabling biomechanical measurement and simulation across workflows and software applications

\section{INTRODUCTION}

Biomechanical measurement and simulation have many uses across medicine, sports, and everyday life for improving performance and health. Understanding of how people and animals move has led to improved fundamental anatomical analysis (Muybridge, 1887), sporting technique (Cohen et al., 2015, 2018, 2017; Harrison et al., 2019, 2016), physiotherapy interventions, elderly care (Pogorelc et al., 2012), and orthopaedic surgical design (Stebbins et al., 2010). Computational simulation of body movement has been used to improve understanding of neuromuscular function during normal activities (Anderson and Pandy, 2001, 1993), joint loading (Crowninshield et al., 1978), and surgical outcomes (Delp et al., 1990; Heller et al., 2001). However, both the measurement and simulation of human movement have traditionally required the use of expensive hardware and software and high technical expertise, limiting the scale and breadth of their use.

Recently these tools have become more accessible (and potentially more widely useful) due to the opensourcing of biomechanics software (Delp et al., 2007) and musculoskeletal models (https://simtk.org), the development of cheap camera hardware (Pfister et al., 2014), and advances in machine learning (Cao et al., 2018). Research-quality musculoskeletal models can be accessed free-of-charge and used to investigate body kinematics and loading (e.g. Hamner et al., 2010).

A remaining barrier to entry for wider use by scientists, clinicians and the wider public of biomechanical measurement and simulation is the technical expertise required to customise and apply existing software and hardware. Software tools such as OpenSim (Delp et al., 2007) require coding knowledge are therefore not accessible for all scientists to construct and apply models and are limited in the range of environmental interactions that can be modelled. The custom use of OpenPose code or Kinect sensors is similarly constrained by needs for specific technical expertise.

Workspace is a scientific workflow system that simplifies the use, interaction with, and customisation of disparate code-bases for modular experimental use or license-able standalone deployment (Bolger et al., 2016, 2015; Cleary et al., in review; Watkins et al., 2017). Users can combine the strengths of libraries such as Python, linear algebra solvers, and fluid dynamics solvers with in-built capabilities, and can interact with workflow inputs and outputs using intuitive widgets. Using Workspace encourages and supports the use of good software architecture that unifies contributions from multiple researchers or teams into a common platform. Users can develop plugins that introduce new capabilities and widgets that can be shared with coworkers, collaborators and customers with less time spent on software infrastructure.

In this paper we introduce the Bio-Mechanic (BM) plugin built in Workspace and demonstrate the utility of the plugin to address a range of needs in biomechanical science including in a commercial research environment. The scientific background of the code base is summarised, the links to external libraries are discussed, and use cases are described. The benefits of developing the plugin are presented and linked to the design principles of Workspace.

\section{STRUCTURE OF THE BM WORKSPACE PLUGIN}

The BM plugin is being developed at the CSIRO and is currently being expanded with a focus on greater utility by non-technical users, volume of use, and easier model customisation. Whilst originally built for sports applications, the developers are creating use cases for workplace scenarios, such as for reducing manual handling injury, and health care scenarios, such as for safety improving ageing in the home.

A schematic of the BM plugin is shown in Figure 1. A typical analysis involved:

- Development of a personalised body model using a combination of marker data or video data and either anthropomorphic measurements (e.g. height, weight, arm/length lengths etc) or laser body scanning

- Specification of joint and centre of mass motions using marker time-series data or video and markerless motion capture

- Model refinement (if required) which may involve the removal of artificial noise from movement signals or improvements to model parameters from the analysis of simulation outputs

- Forward dynamics simulation of body movements and the interaction of the body with its environment. This may include muscle dynamics and internal body loading.

- Analysis of body motions and simulation outputs

- Graphing and tabulating of movement and simulation data for reporting 
Harrison and Cohen, The Bio-Mechanic Workspace plugin: enabling biomechanical measurement and simulation across workflows and software applications

- $3 \mathrm{D}$ visualisation of movements and simulation results including external and internal forces and the predicted stresses or flows of components of the environment (e.g. water flows and or ground responses to impact).

- $\quad$ Output of models and results for sharing with collaborators (e.g. skeletal formats such as BVH or OpenSim), further analysis (e.g. statistical analysis using R), or visualisation using other software (e.g. Maya)

This analysis can be easily performed using BM operations in Workspace, either as a custom-built workspace, shared as a pre-built workflow, or packaged as a licensed standalone software.

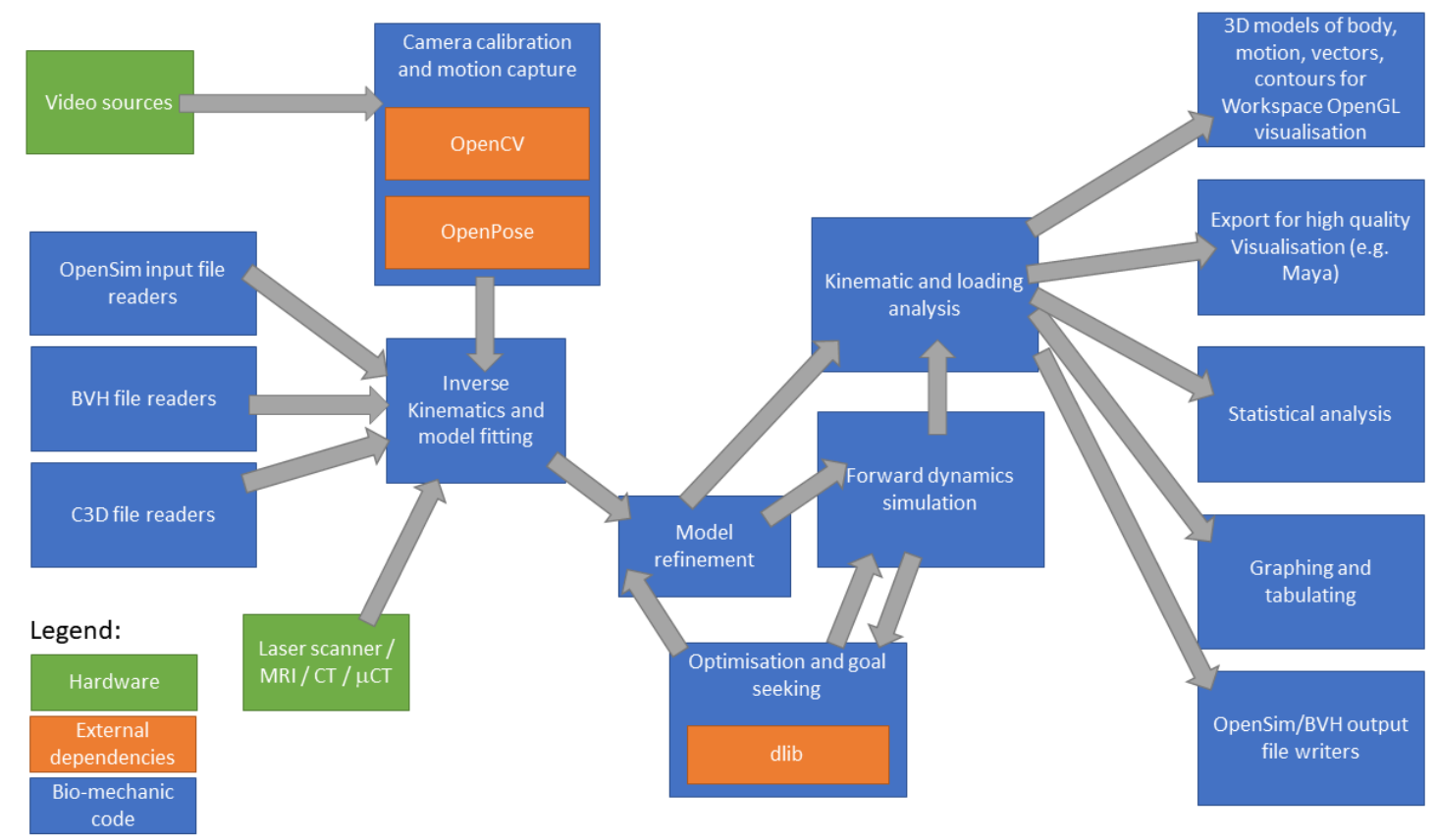

Figure 1. schematic of the BM plugin for Workspace. The flow of data from external sources through analysis and outputs is shown from left to right. The legend is shown in the bottom left corner.

\section{USE CASES}

Uses of the BM plugin range from simple conceptual experimentation by technical users to deployed standalone applications that can be used by non-technical members of the public. Here we describe four example use cases (E1 - E4).

\section{1. $\quad$ E1 - Visualisation of moving people using freely available models and data}

Figure 2a shows a simple example workflow that uses operations from the BM plugin. A published musculoskeletal model and motion dataset (Dorn et al., 2012) is read into the workflow using the operations "OpenSim Skeleton Reader" and "OpenSim Motion Reader". Data from one time instance is visualised by selecting a frame of the motion data. The transformations of each joint centre are calculated using a modular operation. Finally, the mesh models of the skeleton and muscles are constructed in separate operations. The mesh models are connected to the built-in "Visualise model" workflow which, when connected to scene widget, gives the 3D render shown on the right side of Figure 2a. Panels b-d of Figure 2 show the visualisation of the musculoskeletal model at frames 10,30, 50 and 70 of the motion data. This simple usecase demonstrates how a useful and re-usable workflow with a small number of elements can be built and shared with collaborators.

\subsection{E2 - Visualisation of results from a coupled biomechanical-smoothed particle hydrodynamics (B-SPH) model}

The BM plugin has been used to build, visualise, and analyse results for coupled B-SPH models. In the case of simulation of K1 kayaking (Harrison et al., 2019), this involved:

1. Model set up:

a. The construction of an array of 37 million SPH particles 
Harrison and Cohen, The Bio-Mechanic Workspace plugin: enabling biomechanical measurement and simulation across workflows and software applications

b. Pre-processing and specification of mesh models the kayak, paddle and person

c. Specification of a skeletal model of the athlete.

2. Model analysis and post-processing:

a. Surface mesh construction for the fluid phase

b. Calculation of values of external body force and joint torque

3. Visualisation and reporting

a. Visualisations of model set ups, as shown in Figures 1,3 and 5 of Harrison et al. (2019).

b. Visualisations of model results, as shown in Figure 6 of Harrison et al. (2019)

c. Calculations of net force and torque on the athlete's body

The effort required to perform the complex model set up, analysis and reporting was reduced substantially using the BM plugin and existing workflows.

(a)

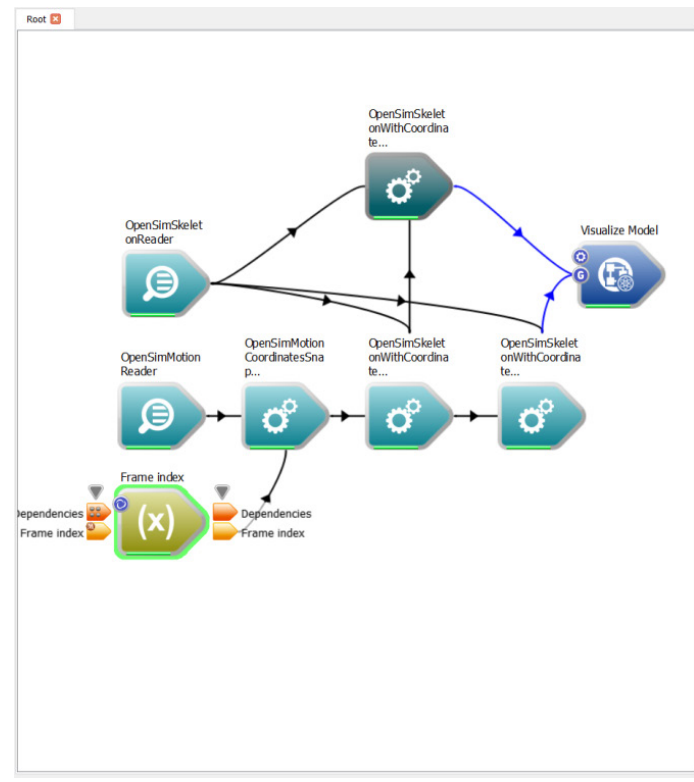

(b)

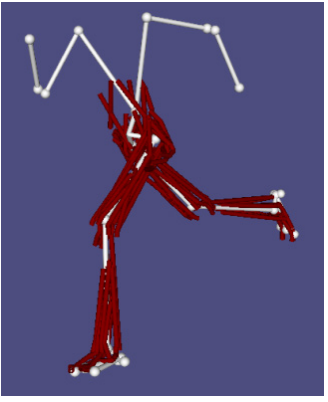

(c)

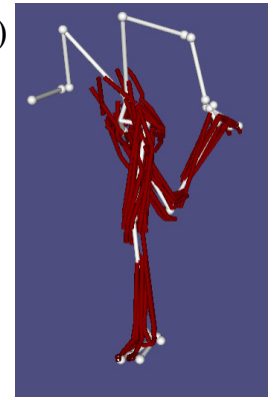

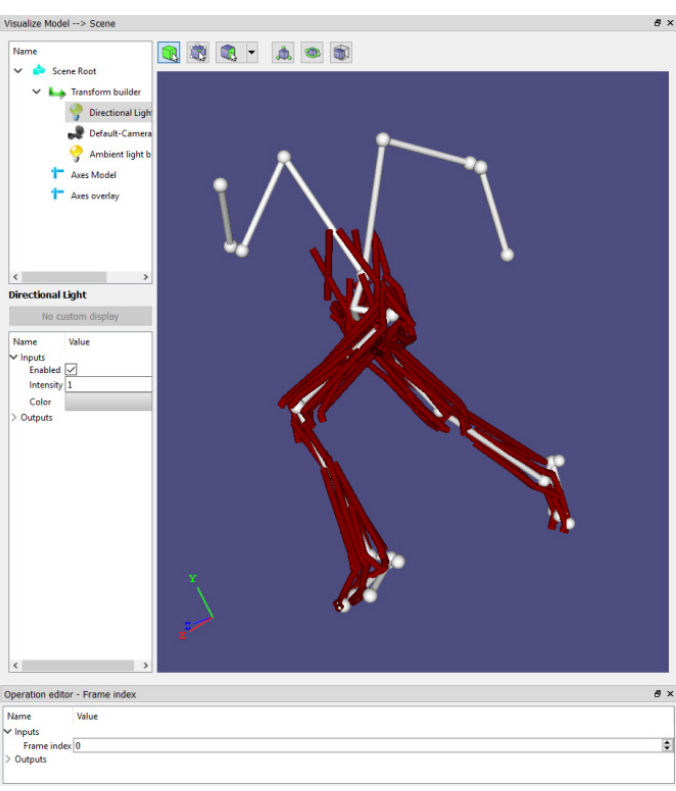

(d)



(e)

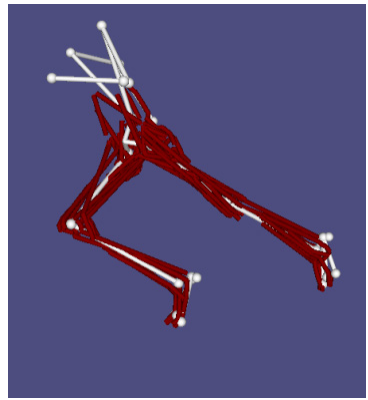

Figure 2. Visualisation of a human running model using a simple BM enabled workflow using data from Dorn et al. (2012). The workflow (a) involves six BM operations, one variable (yellow icon) and one in built Workspace workflow (blue icon). The running technique (b-e) is easily visualised by interactive modification of the frame index variable.

\subsection{E3 - Simulation of the interactions with the external environment using the Rigid Body Dynamics solver}

Forward dynamics simulation of exercise is one of the most common and useful types of biomechanical simulation, but these remain difficult to perform by non-expert users. A walking model and data from Delp et al. (1990) were used in combination with the BM operation called "Rigid Body Dynamics" to simulate overground walking. The simulation workflow is shown in Figure 3. File readers for the skeleton, skin surface, skin rigging, and skeletal motion populate the human body model. A "Ground" datatype is built using the inbuilt Workspace "Compose Group" operation. Simulation controls are intuitively prescribed using the widget for the "Rigid Body Dynamics" operation. Numerical results such as centre of mass motion and external (ground reaction) force are plotted in the interactive plotting widget. A video of the 3D visualisation of simulation results is created once the simulation is complete. For this use case the workflow 
Harrison and Cohen, The Bio-Mechanic Workspace plugin: enabling biomechanical measurement and simulation across workflows and software applications

is simple, easy to construct, general for use, shareable and able to be combined with a user interface into standalone software as required.

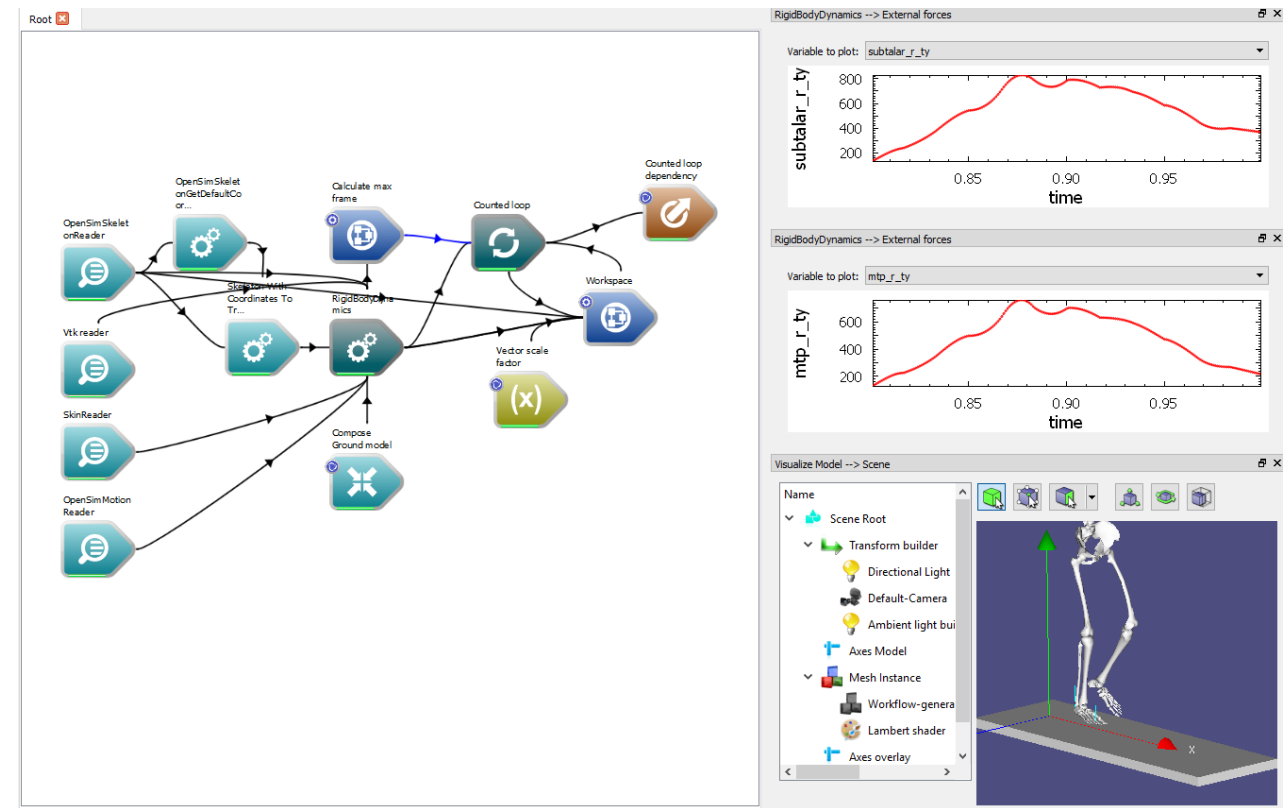

Figure 3. Workflow for forward dynamics of human walking. Four file reader operations, three pre-processing operations and four post processing operations are combined with the solver to simulate walking. Force results (top right) and a visualisation (bottom right) are immediately available when the solver operation is complete.

(a)

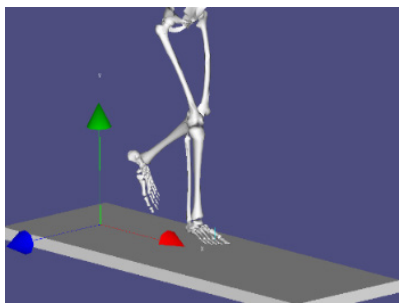

(d)

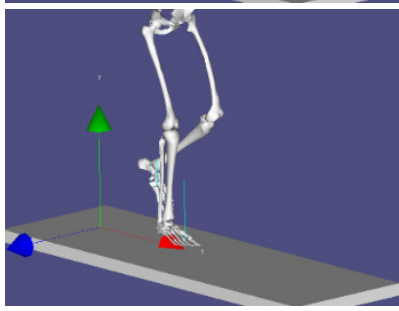

(b)

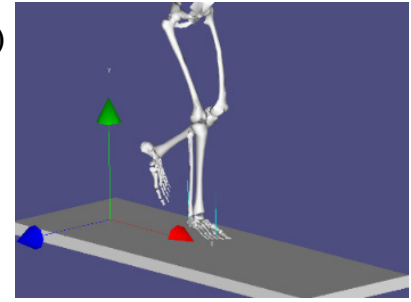

(e)

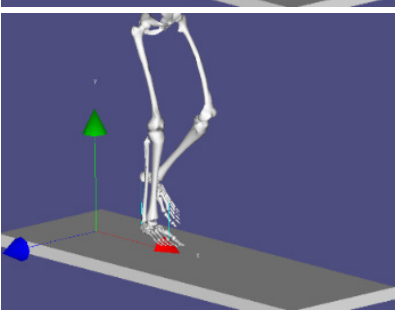

(c)

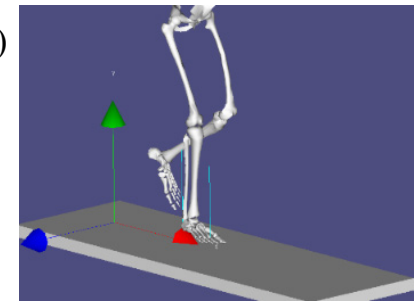

(f)

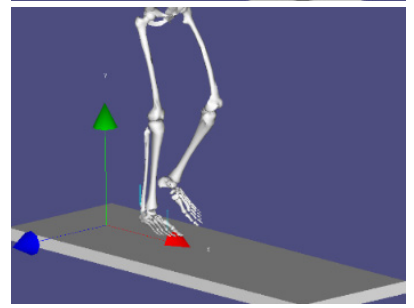

Figure 4. Results of the dynamic simulations of human walking from (a) heel strike through to (f) end of stance.

Figure 4 shows example results of the forward dynamics simulation of human walking. As the right leg extends downwards the foot makes contact with the ground. After contact is made the ground reaction forces (shown as blue lines from the front and rear of the foot) increase in magnitude and is generally aligned upwards. As a result, the centre of mass is accelerated upwards and the forwards speed is reduced modestly. At the end of this stance phase the foot pushes off the ground and the GRF is orientated forwards, accelerating the centre of mass forwards. This type of simulation can be extended to more complicated movements, to include internal body forces, for multiple people or animals, and for more complex environmental interactions.

\section{4. $\quad$ E4 - Standalone software for the virtual experimentation of elite diving}

Dive Mechanic is a standalone software application that was built upon the BM plugin for Diving Australia to allow coaches and athletes to virtually experiment with diving technique before implementing changes to physical training (Cohen et al., 2017). It was developed using less than one full time equivalent year of work 
Harrison and Cohen, The Bio-Mechanic Workspace plugin: enabling biomechanical measurement and simulation across workflows and software applications

which was possible because of the productivity benefits derived from the plugin design and the Workspace environment. The software combines laser scan models of five athletes with more than sixty digitised motions of springboard diving performance. Users can visualise any of the dives from the database and also modify the take-off conditions and pose change timings of each dive. The software was primarily designed with the aim to provide national level coaches with the ability to virtually experiment with the timing of key events in the dives and improve the timing of synchronised pairs. Australian coaches were able to use the software with athletes without any training in the lead up to the 2016 Olympic games, thereby demonstrating the intuitive nature of the software for non-technical users.

\section{BENEFITS OF DEVELOPING IN THE WORKSPACE WORKFLOW ENVIRONMENT}

Significant effort is invested in software development in the area of biomechanics but much of the outputs of such effort is not reusable or not sharable code. Few research groups have the resources to convert their code into interactive commercialised standalone software. Workspace has four design principles (Analyse, Collaborate, Everywhere, Commercialise) that seek to address common productivity and deployment issues in the scientific software domain (Bolger et al., 2016, 2015, 2015; Watkins et al., 2017). There are several clear benefits that result from the decision to develop the capabilities in the BM plugin in the Workspace framework:

- Interactivity and intuitiveness. Each operation input is automatically assigned a widget that allows interactivity during the running of a workflow. The visual interface is very intuitive and has been proven to be useable by non-technical users. For instance, the Dive Mechanic application (E4) was provided to coaches and athletes without training and they were able to immediately use the software to virtually experiment with elite diving technique (Cohen et al., 2017).

- Interoperability and reusability. All unit operations are modular by design and use a common interface and typically common datatypes. They are immediately interoperable without any extra coding effort. Operations and workflows are therefore reusable and easily shareable. All four of the example use cases (E1-4) make use of common datatypes, widgets, file IO operations, skeletal pose calculation operations and visualisation tools that can be re-arranged easily for novel uses and applications.

- Linkages with other code bases and solvers. Workspace is already linked to libraries such as Python, R, Matlab, VTK and can read file formats such as STL, IGES, OBJ, C3D, BVH, and most image and video formats. Examples 1-4 all make use of the VTK library for storing mesh data and E2 made use of the BVH format. We have linked the BM plugin to the CSIRO SPH-DEM solvers (which are Fortran-based) easily (as shown in E2) and many other similar linkages have been made by other teams.

- Commercialisation and speed to market. Software deliverables can be built from conceptual stage to production stage very naturally, experimented with and reconfigured quickly, tested for user experience and attached to user interfaces very easily. The Dive Mechanic application (E4) was able to be developed in a relatively short amount of time because of the reusability of the code base, the ability to quickly experiment with design, and the in-built interactivity of user interface widgets. Software designers can move from prototype product back to workflow seamlessly to re-evaluate design or to make minor changes. Professional, licensed software applications can be generated by users with strong biomechanical domain skills but without strong computer science backgrounds.

- Productivity. A strong case for improved productivity of code design, creation and utilisation is described in the aforementioned benefits. Example use cases E1 and E3 can be built in less than ten minutes for novel applications of the plugin capabilities. Design, development and deployment of E4 was substantially more productive for the reasons listed above. Workflows are frequently re-used and shared and can be more quickly and easily modified for new usage than normal code in most cases.

\section{CONCLUSIONS}

The Bio-Mechanic (BM) Workspace plugin is a full-featured codebase for performing biomechanical measurement and simulation, whether in small conceptual experiments, complex shareable and interactive workflows, or detailed and licensed standalone software applications. Because it has been developed in the Workspace environment a range of benefits are available and have been demonstrated by this plugin including intuitiveness of interactivity, interoperability and reusability of IP, ease of linkages with other codebases and solvers, ease and speed of commercialisation and increased productivity. Custom use of the plugin is not limited to code developers as is the case for other biomechanical software packages such as 
Harrison and Cohen, The Bio-Mechanic Workspace plugin: enabling biomechanical measurement and simulation across workflows and software applications

OpenSim (Delp et al., 2007) and OpenPose (Cao et al., 2018). Example use cases are described that demonstrate (1) ease of construction of bespoke workflows for visualisation and simulation, (2) the ability to post process complex simulation data for scientific reporting, and (3) quick deployment of detailed scientific software to non-technical users.

\section{REFERENCES}

Anderson, F.C., Pandy, M.G., 2001. Static and dynamic optimization solutions for gait are practically equivalent. J. Biomech. 34, 153-161.

Anderson, F.C., Pandy, M.G., 1993. Storage and utilization of elastic strain energy during jumping. $J$. Biomech. 26, 1413-1427.

Bolger, M., Cleary, P.W., Cohen, R.C.Z., Harrison, S.M., Hetherton, L., Rucinski, C., Sankaranarayanan, N., Thomas, D., Watkins, D., Zhang, Z., 2016. Workspace: a fast and low cost methodology for delivering commercial applications based on Research IP. Presented at eResearch Australasia, Melbourne, Australia.

Bolger, M., Cleary, P.W., Heatherton, L., Rucinski, C., Thomas, D., Watkins, D., 2015. Workspace: Scientific Workflows and Applications for multiple Environments. Presented at the eResearch Australasia Conference, Brisbane, Australia.

Cao, Z., Hidalgo, G., Simon, T., Wei, S.-E., Sheikh, Y., 2018. OpenPose realtime multi-person 2D pose estimation using Part Affinity Fields, in: ArXiv Preprint ArXiv1812.08008.

Cleary, P.W., Thomas, D., Hetherton, L., Bolger, M., Hilton, J.E., Watkins, D., in review. Workspace: a workflow platform for supporting development and deployment of modelling and simulation. Math. Comput. Simul. in review.

Cohen, R.C., Cleary, P.W., Mason, B.R., Pease, D.L., 2015. The role of the hand during freestyle swimming. J. Biomech. Eng. 137, 111007.

Cohen, R.C.Z., Cleary, P.W., Mason, B.R., Pease, D.L., 2018. Forces during front crawl swimming at different stroke rates. Sports Eng. 21, 63-73.

Cohen, R.C.Z., Harrison, S.M., Cleary, P.W., 2017. Dive Mechanic: Bringing 3D virtual experimentation to elite level diving using the Workspace workflow engine. Presented at MODSIM 2017, Hobart, Australia.

Crowninshield, R.D., Johnston, R.C., Andrews, J.G., Brand, R.A., 1978. A biomechanical investigation of the human hip. J. Biomech. 11, 75-85.

Delp, S.L., Anderson, F.C., Arnold, A.S., Loan, P., Habib, A., C. T. John, E. Guendelman, D. G. Thelen, 2007. OpenSim: Open-Source Software to Create and Analyze Dynamic Simulations of Movement. IEEE Trans. Biomed. Eng. 54, 1940-1950.

Delp, S.L., Loan, J.P., Hoy, M.G., Zajac, F.E., Topp, E.L., Rosen, J.M., 1990. An interactive graphics-based model of the lower extremity to study orthopaedic surgical procedures. IEEE Trans. Biomed. Eng. 37, 757-767.

Dorn, T.W., Schache, A.G., Pandy, M.G., 2012. Muscular strategy shift in human running: dependence of running speed on hip and ankle muscle performance. J. Exp. Biol. 215, 1944-1956.

Hamner, S.R., Seth, A., Delp, S.L., 2010. Muscle contributions to propulsion and support during running. $J$. Biomech. 43, 2709-2716.

Harrison, S.M., Cleary, P.W., Cohen, R.C.Z., 2019. Dynamic simulation of flat water kayaking using a coupled biomechanical-smoothed particle hydrodynamics model. Hum. Mov. Sci. 64, 252-273.

Harrison, S.M., Cohen, R.C.Z., Cleary, P.W., Barris, S., Rose, G., 2016. A coupled biomechanical-Smoothed Particle Hydrodynamics model for predicting the loading on the body during elite platform diving. Appl. Math. Model. 40, 3812-3831.

Heller, M.O., Bergmann, G., Deuretzbacher, G., Dürselen, L., Pohl, M., Claes, L., Haas, N.P., Duda, G.N., 2001. Musculo-skeletal loading conditions at the hip during walking and stair climbing. J. Biomech. 34, 883-893.

Muybridge, E., 1887. Animal locomotion. Da Capo Press.

Pfister, A., West, A.M., Bronner, S., Noah, J.A., 2014. Comparative abilities of Microsoft Kinect and Vicon 3D motion capture for gait analysis. J. Med. Eng. Technol. 38, 274-280.

Pogorelc, B., Bosnić, Z., Gams, M., 2012. Automatic recognition of gait-related health problems in the elderly using machine learning. Multimed. Tools Appl. 58, 333-354.

Stebbins, J., Harrington, M., Thompson, N., Zavatsky, A., Theologis, T., 2010. Gait compensations caused by foot deformity in cerebral palsy. Gait Posture 32, 226-230.

Watkins, D., Thomas, D., Hetherton, L., Bolger, M., Cleary, P.W., 2017. Workspace - a Scientific Workflow System for enabling Research Impact, in: 22nd International Congress on Modelling and Simulation (MODSIM 2017). Hobart, Tasmania, Australia. 